OPEN ACCESS

Edited by:

Nebojša Trajković, University of Niš, Serbia

Reviewed by: Josie Booth,

The University of Edinburgh,

United Kingdom

Danilo Radanovic,

University of Novi Sad, Serbia

*Correspondence:

Shawnda A. Morrison shawnda.morrison@fsp.uni-lj.si

Specialty section: This article was submitted to

Health Psychology, a section of the journal

Frontiers in Psychology

Received: 17 October 2021 Accepted: 11 January 2022 Published: 08 February 2022

Citation: Sember V, Jurak G, Starc $G$ and Morrison SA (2022) Can Primary School Mathematics Performance Be Predicted by Longitudinal Changes in Physical Fitness and Activity Indicators?

Front. Psychol. 13:796838 doi: 10.3389/fpsyg.2022.796838

\section{Can Primary School Mathematics Performance Be Predicted by Longitudinal Changes in Physical Fitness and Activity Indicators?}

\author{
Vedrana Sember, Gregor Jurak, Gregor Starc and Shawnda A. Morrison*
}

Faculty of Sport, University of Ljubljana, Ljubljana, Slovenia

Objective: To determine to what extent physical fitness indicators and/or moderate to vigorous physical activity (MVPA) may account for final mathematics academic performance $\left(A P_{\text {math }}\right)$ awarded at the end of primary school.

Methods: School-aged youth were sampled in a repeated-measures, longitudinal design in Grade 6 ( $\sim 11$ years), and again in Grade 9 ( $\sim 14$ years). The youth $(N=231$, 111 girls) completed a fitness test battery consisting of: flamingo balance test, standing long jump, backward obstacle course, plate tapping, sit ups, sit and reach, handgrip, and 20-m shuttle run. AP math scores were obtained for all children at the end of Grade 5, end of Grade 8, and end of Grade 9 (their final year of primary school). In a sub-sample of Grade 6 youth ( $N=50,29$ girls), MVPA was measured objectively via SenseWear Pro Armbands (MVPABB for seven consecutive days, with measurements repeated in Grade 9.

Results: Math scores decreased from Grade 6 to 9 for both boys and girls $(95 \% \mathrm{Cl}$ : -0.89 to $-0.53, p<0.001)$. MVPAOB was reduced by $\sim 45.7$ min $(-33 \%)$ from Grade 6 to $9(p<0.01)$. Significant main and interaction effects are noted for each fitness indicator $(\rho<0.05)$. A backward stepwise multiple regression analysis determined significant shared variance in final $\mathrm{AP}_{\text {math }}$ grade to the change scores from Grade 6 to Grade 9 in: $\Delta \mathrm{AP}_{\text {math }}, \Delta$ backward obstacle course, $\Delta$ sit and reach, and $\Delta$ sit-ups $\left[R^{2}=0.494, F(4,180)=43.67, p<0.0001\right]$. A second regression was performed only for the youth who completed MVPA $A_{O B}$ measurements. In this sub-sample, MVPA $A_{O B}$ did not significantly contribute to the model.

Conclusion: Longitudinal changes in youth fitness and their delta change in $\mathrm{AP}_{\text {math }}$ score accounted for $49.4 \%$ of the variance in the final math grade awarded at the end of Grade 9. Aerobic power, upper body strength, and muscular endurance share more common variance to final math grade in boys, whereas whole-body coordination was the more relevant index in girls; this finding suggests that future research exploring the relationship of $\mathrm{AP}$ and $\mathrm{PF}$ should not be limited to cardiorespiratory fitness, instead encompassing muscular and neuro-muscular components of PF.

Keywords: accelerometers, physical fitness surveillance, paediatric exercise, health-related risk factors, population health, arithmetic, children 


\section{INTRODUCTION}

Physical activity (PA) is a dynamic state of being (Reid et al., 2019) which can be defined as any bodily movement produced by skeletal muscles resulting in energy expenditure (Caspersen et al., 1985) greater than that which exceeds hibernation (Fletcher et al., 1996). Participating in regular PA is indispensable to maintaining a healthy lifestyle, in part because of the concomitant positive impacts on skeletal (Gunter et al., 2012), metabolic (Janssen and LeBlanc, 2010), cardiovascular (Fernhall and Agiovlasitis, 2008), and psychosocial functioning of the human body (Biddle and Asare, 2011). Attaining low levels of $\mathrm{PA}$ and the attendant poor aerobic fitness is associated with declines in academic performance (AP) (Chaddock et al., 2011), the deterioration of brain structures, cognitive abilities, and general brain function (Sibley and Etnier, 2003; Castelli et al., 2007; Chaddock et al., 2010; Donnelly et al., 2016; GarcíaHermoso et al., 2021). Increasing PA has therefore, long been suggested to exert a positive impact on AP, especially since learning complex movements stimulates the frontal cortex of the brain, which is also active in the learning and problemsolving process (Daly-Smith et al., 2018; Singh et al., 2019; Duffey et al., 2021).

There is a growing body of work which have found significant relationships between PA and AP (Chaddock-Heyman et al., 2013; Daly-Smith et al., 2018; Sember et al., 2020b). However, the positive effects of PA on AP may accrue most readily only when there is an adequate amount of vigorous PA included (Tomporowski et al., 2011; Phillips et al., 2015). Evidence regarding the association between $\mathrm{PA}$ and any broader aspect(s) of AP have (thus far) remained ambiguous, with some researchers also finding negative, or null effects in this relationship (Coe et al., 2006; Beck et al., 2016; Riley et al., 2016). These inconsistent findings may be due to a difficulty in precisely assessing both the overall amount, and intensity of, PA, which children and adolescents regularly undertake (Monyeki et al., 2018; Sember et al., 2020a).

The level of physical fitness (PF) for a given individual is related to a great many factors, including the outcome of their habitual PA habits, genetics, socio-economic status, and environment, among others. Physical fitness can be highly informative when examining the health effects of PA and AP (Sardinha et al., 2014), including recent evidence that cardiorespiratory fitness is associated with larger brains during a critical phase in children, when the brain is growing (Cadenas-Sanchez et al., 2020). For example, it has been shown that after-school PA improves children's cardiovascular endurance, which then mediates enhancements in AP (Fredericks et al., 2006). A meta-analysis (including articles from 2005 to 2015), which investigated the relationship between PF and AP asserted that cardiorespiratory fitness, speed-agility, motor coordination, and perceptual-motor skills are each highly associated fitness indicators to AP (RuizAriza et al., 2017). Moreover, several studies have identified positive relationships between cardiorespiratory fitness, body mass and AP (Eveland-sayers et al., 2009; Van Dusen et al., 2011; Rauner et al., 2013; Torrijos-Niño et al., 2014) or overall
PF (Starc et al., 2017). Findings on the relationship of AP to strength and flexibility remain unclear (Chaddock-Heyman et al., 2014; Tannehill et al., 2015; Ruiz-Ariza et al., 2017; Esteban-Cornejo et al., 2019).

Although there is an abundance of scientific papers dealing with the relationship between $\mathrm{PA}$ and cognitive function, researchers often use indirect assessments of PA in their work. Indeed, although researchers and public health policies continue to cite PA as a primary movement/health-based indicator, it is also a highly individual one, where any two individuals may need varying doses (i.e., frequency, duration, intensity, and type of activity) to achieve the same physiological and/or health related benefits as one another (Reid et al., 2019). Notably, objective PA assessment is often not feasible for large studies involving hundreds or even thousands of participants, due to its high cost and complications arising from extracting PA data from the measuring devices (Dowd et al., 2018). Although several studies have found a significant relationship between aerobic function and AP, most have not considered longitudinal changes in PA and $\mathrm{PF}$ across their sample.

Thus, the purpose of this study was four-fold: (1) to determine longitudinal changes in PF, MVPA (measured objectively) and $\mathrm{AP}_{\text {math }}$ in a cohort of Slovenian youth across a 3-year timespan, (2) to determine whether individual PF indicators were correlated to MVPA or $\mathrm{AP}_{\text {math }}$ scores within a given Grade, (3) to determine whether changes in MVPA or PF indicators related to changes observed in $\mathrm{AP}_{\text {math }}$, and (4) to what extent could these changes over time account for final $\mathrm{AP}_{\text {math }}$ score attained at the end of primary school. It was hypothesized that youth who were able to maintain higher PA and PF would maintain or achieve higher math scores at the end of their schooling period compared to those who were less active or fit.

\section{MATERIALS AND METHODS}

\section{Research Design and Study Sample}

We obtained the data for this longitudinal, cross-sectional study within The Analysis of Children's Development in Slovenia (ACDSi), an ongoing study used to monitor the physical and motor development of children and youth in Slovenia every 10 years for the last five decades (Jurak et al., 2013; Starc et al., 2015). The ACDSi study was approved by the Slovenian National Medical Ethics Committee (ID:138/05/13), following the Declaration of Helsinki. We obtained written, informed, parental consent and child assent before testing for each child involved in the study. Children and youth participated voluntarily and anonymously and had the option of freely withdrawing from the measurements at any time. The ACDSi study uses a sentinel approach to provide a nationally representative sample of children from 11 different locations, stratified according to their environment (e.g., village, town, industrial town, and city). The primary sampling unit is the school, and the secondary one defined as the schoolchildren's class. At each of the 11 primary school locations, research teams were divided into three test stations: anthropometry, motor and aerobic fitness and psychology. 
The research team from the Faculty of Sports, University of Ljubljana collected data for this study in autumn 2013, then $\sim 3$ years later during summer of 2016, and again in autumn 2016. In the 2013 generation of the ACDSi study, the Grade 6 sample included 231 youth (120 boys and 111 girls), aged 11 years old (born on October 01, 2002, \pm 6 months). The average age of all youth included in the Grade 6 sample was $11.29 \pm 0.30$ years (boys $11.3 \pm 0.32$; girls $11.29 \pm 0.27$ ), and the average age of the youth wearing SWA was $11.23 \pm 0.3$ years (boys $11.24 \pm 0.36$; girls $11.22 \pm 0.25)$. All participants were measured again, exactly 3 years later, in Grade 9.

\section{Experimental Measures \\ Physical Activity}

We assessed MVPA objectively on a sub-sample of children $(N=50$, boys $=21$ girls $=29)$ wearing a multi-sensor device (SWA, Bodymedia SenseWear Pro Armband; BodyMedia Inc., Pittsburgh, PA, United States, MVPAOB $)$. Device specifications, data collection techniques, and standard methodology is outlined in detail elsewhere (Sember et al., 2020a). The SWA device is based on the recognition of energy expenditure patterns resulting in an estimation of $\mathrm{PA}$ and is considered to be a reliable measuring device in children and youth (Calabró et al., 2009; Soric et al., 2013; Stålesen et al., 2016). Participants wore the SWA on their triceps (i.e., right side of upper arm) for 1 week (Cain et al., 2013), 24 h per day, except when they were showering, bathing, or performing water-based, or sporting activities (e.g., swimming and/or competitions in other sports).

\section{Physical Fitness}

We assessed PF via the following indicators: $20-\mathrm{m}$ shuttle run (peak aerobic power, $\dot{\mathrm{V}} \mathrm{O}_{2 \text { peak }}$ ), polygon backward obstacle course (coordination), handgrip (muscular strength), standing long jump (power), flamingo test (balance), sit-ups (repetitive strength) plate tapping (reaction time) and sit and reach (hip joint flexibility). Details on test protocols are available elsewhere (Jurak et al., 2013; Starc et al., 2015). $\dot{\text { VO }} 2$ peak was estimated using Mahar's prediction equation (Mahar et al., 2011), estimated from the 20-m multistage shuttle run performance test, which was conducted following Leger's original test protocol (Leger and Lambert, 1982). Jamar's dynamometer (TEC, Clifton, NJ, United States) was used to measure handgrip strength on the child's dominant hand.

\section{Mathematics Performance}

The indicator taken to best reflect AP in Slovenian schoolchildren and youth was their math grade, awarded by their teachers at the end of the previous academic year. Math grades were recorded for all youth representing the start of Grade 6 (i.e., final score awarded at end of Grade 5), the start of Grade 9 (final score awarded at end of Grade 8), and their final mathematics mark, awarded at the end of Grade 9. The math grade represents a modest indicator of overall AP in the literature, but it is especially appropriate for the Slovenian setting, with an $r=0.50$ (Flere et al., 2009) and higher levels of reliability (0.89-0.94) (Carlson et al., 2008) than other subjects or language scores (e.g., Slovenian or English), for example. In Slovenia, math grades are awarded following next-order methodology: 1 (inadequate), 2 (sufficient), 3 (good), 4 (very good) and 5 (excellent).

\section{Data Processing}

Objective physical activity (MVPA $\mathrm{OB}$ ) assessment of moderateto-vigorous physical activity (MVPA) were analyzed using the Bodymedia SenseWear Professional 8.1 Software package and scored/adjusted manually where necessary. Microsoft Excel 2007 was used for removing artifacts, counts $>16,000$, constant values $\geq 0$, and sequences of zeroes, where a sequence was defined as 20 or more zeroes. MVPA ${ }_{O B}$ data was processed with BodyMedia SenseWear Armband software 3.0 (standardized for accelerometer SenseWear Armband), following the 70/80 rule (Catellier et al., 2005) and non-wear time within day (Troiano et al., 2008). We only included the data of participants who wore the SWA device at least 5 days in a row, including both weekend days, and whose wear time exceeded 90\%, following methods described elsewhere (Cain et al., 2013). The SWA data was collected in 1-min epoch intervals. The main rationale for using this conservative approach was to reduce the error of under- and over-estimation of PA due to missing wear-time, often an issue in current PA child health studies. Finally, difference scores of fitness and activity indices were calculated between Grade 6 and Grade 9 before statistical analyses were conducted.

\section{Statistical Analyses}

We conducted all statistical analyses using SPSS version 27.0 (IBM Inc., Armonk, NY, United States). Data are presented as means and standard deviation, with 95\% confidence intervals (CI), F-ratios and effect size where appropriate. We determined normality of distribution using the Kolmogorov-Smirnov test and Shapiro-Wilk test. Data were checked for multicollinearity and normality. Longitudinal changes in physical fitness and activity levels from Grade 6 to Grade 9 were compared using a two-way repeated-measures analysis of variance (RM ANOVA), with one within-subjects factor (time, two levels: Grade 6 and Grade 9) and one between-subjects factor (sex, two levels: male, and female), as well as the non-parametric alternative conducted for $\mathrm{AP}_{\text {math }}$ (Friedman test), set at the $p<0.05$ level of significance. Two-tailed, bivariate correlations were run to query whether fitness indicators were related to $\mathrm{MVPA}_{\mathrm{OB}}$ using Pearson's test, calculated for each grade separately. Correlation coefficients between $\mathrm{AP}_{\text {math }}, \mathrm{MVPA}_{\mathrm{OB}}$, and fitness variables were conducted using Spearman's rho $(\rho)$. A backward, stepwise multiple regression was conducted on the entire sample of children to determine to what extent changes in individual fitness indicators shared common variance to final Grade 9 $\mathrm{AP}_{\text {math }}$ scores. The procedure was then conducted separately for the $N=50 \mathrm{MVPA}_{\mathrm{OB}}$ sub-sample with $\triangle \mathrm{MVPA}_{\mathrm{OB}}$ between years as an input variable. Statistical criteria for a given index's inclusion in the model was set at the $p<0.05$ level, and omission 'out' occurred when an index posted $p>0.10$ during the backward step analysis; missing cases were dropped in a listwise fashion. 


\section{RESULTS}

\section{Longitudinal Changes in Physical Activity, Fitness, and Mathematics Academic Performance}

Objectively assessed physical activity $\left(\mathrm{MVPA}_{\mathrm{OB}}\right)$ decreased $\sim 33 \%$ (i.e., $-45.7 \mathrm{~min}$ ) from Grade 6 to Grade 9 [main effect: $F(1,49)=6.225, p=0.016$, Table 1 ], including a significant interaction effect by sex (CI: 10.1-72.2 $\mathrm{min}, p=0.010$ ).

There were significant main effects for time $(p<0.05$, Table 1) in all fitness variables, for both girls and boys. By Grade 9, there were also numerous significant differences between girls and boys within that grade; the 95\% confidence intervals showed that boys produced more aerobic power (measured via shuttle run, CI: $5.9-15.7 \mathrm{~mL} \cdot \mathrm{kg}^{-1} \cdot \mathrm{min}^{-1}$, $p<0.0001$ ), achieved better results in the standing long jump (CI: 8.2-20.7 cm, $p<0.0001$ ), produced more force in handgrip (CI: $0.8-3.3 \mathrm{Nm}, p=0.001$ ), and balanced longer during flamingo testing (CI: 0.1-5.3 s, $p=0.042$ ), whereas girls demonstrated greater hip and lower back flexibility with higher sit and reach scores $(\mathrm{CI}: 5.0-9.6 \mathrm{~cm}$, $p<0.0001)$.

In terms of the longitudinal results of academic performance, assessed by mathematics grade $\left(\mathrm{AP}_{\text {math }}\right)$, this measure decreased from Grade 6 to Grade 9 for all youth (CI: -0.89 to -0.53 , $p<0.0001$ ) equivalent to $\sim 0.7$ of a grade (i.e., $-17 \%)$. The magnitude drop did not differ between boys and girls (CI: -0.32 to $0.04, p=0.120$ ).

\section{Correlations}

\section{Physical Activity and Fitness}

For Grade 6 youth, standing long jump $(r=0.193, p=0.44)$ backward obstacle course $(r=0.208, p=0.030)$, flamingo $(r=-0.232, p=0.015)$ and sit-ups $(r=0.213, p=0.025)$ fitness scores were moderately correlated to MVPA ${ }_{\mathrm{OB}}$ (Table 2). When fitness indicators were separated by sex, fitness variables correlating to MVPAOB differed, with only flamingo test demonstrating a moderate correlation $(r=0.375, p=0.005)$ in boys, and no individual variable reaching significance for girls. By Grade 9, the strength of these linear associations had increased, such that $\mathrm{MVPA}_{\mathrm{OB}}$ was positively correlated to standing long jump $(r=0.305, p=0.002)$, sit-ups $(r=0.288$, $p=0.004)$, handgrip $(r=0.251, p=0.011)$, shuttle run $(r=0.513, p<0.0001)$, and backward obstacle course $(r=0.283$, $p=0.004)$.

\section{Physical Activity and Mathematics Academic Performance}

In Grade 6, mathematics grade $\left(\mathrm{AP}_{\text {math }}\right)$ was negatively correlated to $\mathrm{MVPA}_{\mathrm{OB}}(r=-0.189, p=0.050)$, such that youth who recorded more PA minutes were also more likely to have lower $\mathrm{AP}_{\text {math }}$ scores. This relationship was true only when data were combined. Separated by sex, both boys $(r=0.185, p=0.176)$ and girls $(r=0.193, p=0.161)$ demonstrated no relationship between the variables. $\mathrm{MVPA}_{\mathrm{OB}}$ and $\mathrm{AP}_{\text {math }}$ were not correlated

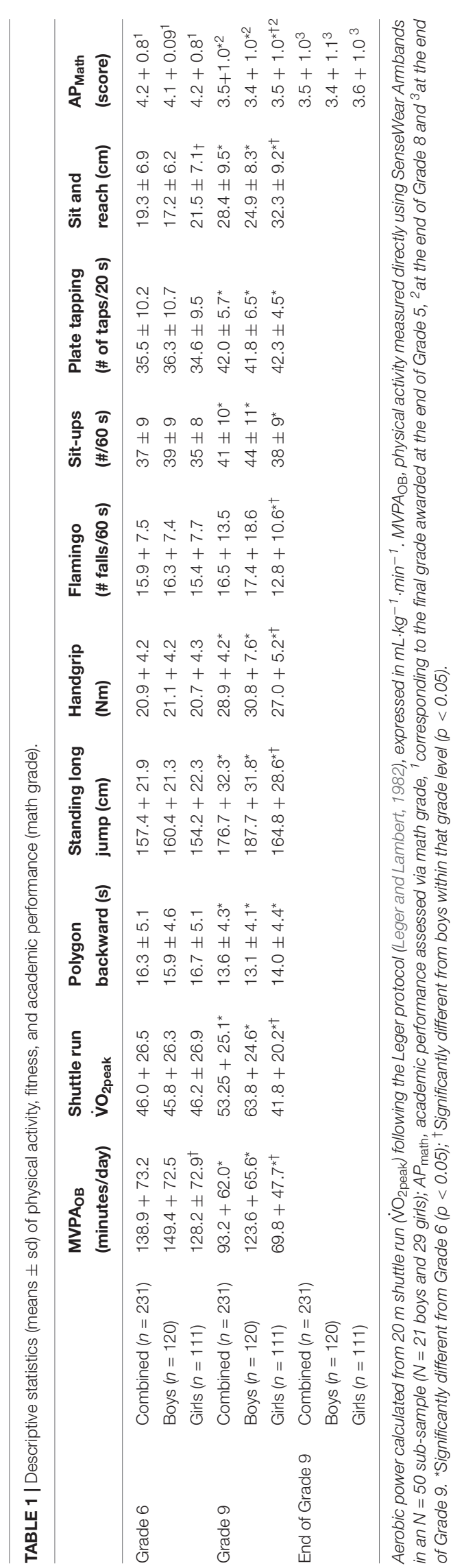


TABLE 2A | Correlation coefficients for dependent measures determined for boys and girls in Grade 6.

\begin{tabular}{|c|c|c|c|c|c|c|c|c|c|c|c|}
\hline Grade 6 & & MVPAOB $_{O}$ & $\begin{array}{l}\text { Shuttle run } \\
\dot{\text { vo }}_{2 \text { peak }}\end{array}$ & $\begin{array}{l}\text { Polygon } \\
\text { backward }\end{array}$ & $\begin{array}{l}\text { Standing } \\
\text { long jump }\end{array}$ & Handgrip & Flamingo & Sit-ups & $\begin{array}{l}\text { Plate } \\
\text { tapping }\end{array}$ & $\begin{array}{l}\text { Sit and } \\
\text { Reach }\end{array}$ & $\mathrm{AP}_{\text {Math }}$ \\
\hline $\mathrm{MVPA}_{\mathrm{OB}}(r)$ & $\begin{array}{l}\text { All } \\
\text { Boys } \\
\text { Girls }\end{array}$ & & & & & & & & & & \\
\hline Shuttle run $\dot{V}_{2 p e a k}$ & $\begin{array}{l}\text { All } \\
\text { Boys } \\
\text { Girls }\end{array}$ & $\begin{array}{l}0.104 \\
0.033 \\
0.188\end{array}$ & & & & & & & & & \\
\hline Polygon backward & $\begin{array}{l}\text { All } \\
\text { Boys } \\
\text { Girls }\end{array}$ & $\begin{array}{l}-0.208^{\star} \\
-0.257 \\
-0.172\end{array}$ & $\begin{array}{l}-0.098 \\
-0.163 \\
-0.064\end{array}$ & & & & & & & & \\
\hline Standing long jump & $\begin{array}{l}\text { All } \\
\text { Boys } \\
\text { Girls }\end{array}$ & $\begin{array}{l}0.193^{\star} \\
0.256 \\
0.102\end{array}$ & $\begin{array}{r}0.043 \\
0.217 \\
-0.073\end{array}$ & $\begin{array}{l}-0.563^{\star \star} \\
-0.727^{\star \star} \\
-0.467^{\star \star}\end{array}$ & & & & & & & \\
\hline Handgrip & $\begin{array}{l}\text { All } \\
\text { Boys } \\
\text { Girls }\end{array}$ & $\begin{array}{l}-0.037 \\
-0.103 \\
-0.004\end{array}$ & $\begin{array}{r}-0.002 \\
0.067 \\
-0.053\end{array}$ & $\begin{array}{l}-0.090 \\
-0.057 \\
-0.114\end{array}$ & $\begin{array}{l}0.207^{\star} \\
0.113 \\
0.276^{\star}\end{array}$ & & & & & & \\
\hline Flamingo & $\begin{array}{l}\text { All } \\
\text { Boys } \\
\text { Girls }\end{array}$ & $\begin{array}{l}-0.232^{\star} \\
-0.375^{\star \star} \\
-0.129\end{array}$ & $\begin{array}{l}-0.200^{*} \\
-0.301^{*} \\
-0,301^{*}\end{array}$ & $\begin{array}{l}0.388^{\star} \\
0.504^{\star \star} \\
0.331^{\star}\end{array}$ & $\begin{array}{l}-0.447^{\star \star} \\
-0.556^{\star \star} \\
-0.405^{\star \star}\end{array}$ & $\begin{array}{l}-0.084 \\
-0.168 \\
-0.021\end{array}$ & & & & & \\
\hline Sit-ups & $\begin{array}{l}\text { All } \\
\text { Boys } \\
\text { Girls }\end{array}$ & $\begin{array}{l}0.213^{*} \\
0.193 \\
0.208\end{array}$ & $\begin{array}{r}0.021 \\
0.182 \\
-0.114\end{array}$ & $\begin{array}{l}-0.420^{\star \star} \\
-0.405^{\star \star} \\
-0.449^{\star \star}\end{array}$ & $\begin{array}{l}0.475^{\star \star} \\
0.444^{\star \star} \\
0.486^{\star \star}\end{array}$ & $\begin{array}{r}0.101 \\
-0.042 \\
0.250\end{array}$ & $\begin{array}{l}-0.378^{\star \star} \\
-0.468^{\star \star} \\
-0.325^{\star}\end{array}$ & & & & \\
\hline Plate tapping & $\begin{array}{l}\text { All } \\
\text { Boys } \\
\text { Girls }\end{array}$ & $\begin{array}{r}0.000 \\
0.038 \\
-0.051\end{array}$ & $\begin{array}{r}-0.034 \\
0.008 \\
-0.084\end{array}$ & $\begin{array}{r}-0.010 \\
-0.138 \\
0.100\end{array}$ & $\begin{array}{c}0.049 \\
0.275^{\star} \\
-0.227\end{array}$ & $\begin{array}{r}-0.045 \\
0.005 \\
-0.164\end{array}$ & $\begin{array}{r}0.060 \\
-0.014 \\
0.109\end{array}$ & $\begin{array}{l}-0.028 \\
-0.003 \\
-0.107\end{array}$ & & & \\
\hline Sit and reach & $\begin{array}{l}\text { All } \\
\text { Boys } \\
\text { Girls }\end{array}$ & $\begin{array}{l}0.085 \\
0.189 \\
0.078\end{array}$ & $\begin{array}{l}0.116 \\
0.033 \\
0.157\end{array}$ & $\begin{array}{l}-0.323^{\star \star} \\
-0.383^{* \star} \\
-0.344^{*}\end{array}$ & $\begin{array}{l}0.086 \\
0.229 \\
0.069\end{array}$ & $\begin{array}{r}0.023 \\
-0.034 \\
0.201\end{array}$ & $\begin{array}{l}-0.180 \\
-0.055 \\
-0.255\end{array}$ & $\begin{array}{l}0.074 \\
0.119 \\
0.133\end{array}$ & $\begin{array}{r}-0.046 \\
0.038 \\
-0.044\end{array}$ & & \\
\hline$A P_{\text {Math }}(\rho)$ & $\begin{array}{l}\text { All } \\
\text { Boys } \\
\text { Girls }\end{array}$ & $\begin{array}{l}-0.189^{\star} \\
-0.185 \\
-0.193\end{array}$ & $\begin{array}{r}0.070 \\
0.177 \\
-0.066\end{array}$ & $\begin{array}{l}-0.183 \\
-0.159 \\
-0.194\end{array}$ & $\begin{array}{l}0.247^{\star \star} \\
0.205 \\
0.294^{\star}\end{array}$ & $\begin{array}{l}0.075 \\
-0.035 \\
0.205\end{array}$ & $\begin{array}{r}-0.104 \\
-0.259 \\
0.099\end{array}$ & $\begin{array}{l}0.169 \\
0.230 \\
0.134\end{array}$ & $\begin{array}{l}-0.140 \\
-0.149 \\
-0.127\end{array}$ & $\begin{array}{r}-0.014 \\
-0.083 \\
0.023\end{array}$ & \\
\hline
\end{tabular}

Aerobic power calculated from $20 \mathrm{~m}$ shuttle run $\dot{\mathrm{VO}}_{2 \text { peak }}$ ) following the Leger protocol (Leger and Lambert, 1982) and expressed in $\mathrm{mL}^{\circ} \mathrm{kg}^{-1} \mathrm{~min}{ }^{-1}$; MVPAOB, physical activity measured directly using SenseWear Armbands in $N=50$ sub-sample ( $N=21$ boys and 29 girls); $A P_{\text {math, }}$ academic performance (math grade, corresponding to the final grade awarded at the end of Grade 5); ${ }^{*}$ Significant correlation $p<0.05$; * ${ }^{*}$ Significant correlation $p<0.01$; Italics font represents non-parametric correlations (Spearman rho). 
TABLE 2B | Correlation coefficients for dependent measures determined for boys and girls in Grade 9.

\begin{tabular}{|c|c|c|c|c|c|c|c|c|c|c|c|}
\hline Grade 9 & & MVPAOB $_{\mathrm{O}}$ & $\begin{array}{l}\text { Shuttle run } \\
\dot{\text { vo }}_{2 \text { peak }}\end{array}$ & $\begin{array}{l}\text { Polygon } \\
\text { backward }\end{array}$ & $\begin{array}{l}\text { Standing } \\
\text { long jump }\end{array}$ & Handgrip & Flamingo & Sit-ups & $\begin{array}{l}\text { Plate } \\
\text { tapping }\end{array}$ & $\begin{array}{l}\text { Sit and } \\
\text { reach }\end{array}$ & $\mathrm{AP}_{\text {Math }}$ \\
\hline $\mathrm{MVPA}_{\mathrm{OB}}$ & $\begin{array}{l}\text { All } \\
\text { Boys } \\
\text { Girls }\end{array}$ & & & & & & & & & & \\
\hline Shuttle run $\dot{V}_{2 p e a k}$ & $\begin{array}{l}\text { All } \\
\text { Boys } \\
\text { Girls }\end{array}$ & $\begin{array}{l}0.513^{\star \star} \\
0.407^{\star \star} \\
0.389^{\star \star}\end{array}$ & & & & & & & & & \\
\hline Polygon backward & $\begin{array}{l}\text { All } \\
\text { Boys } \\
\text { Girls }\end{array}$ & $\begin{array}{l}-0.283^{\star \star} \\
-0.280^{\star \star} \\
-0.358^{\star \star}\end{array}$ & $\begin{array}{l}-0.425^{\star \star} \\
-0.686 \\
-0.248\end{array}$ & & & & & & & & \\
\hline Standing long jump & $\begin{array}{l}\text { All } \\
\text { Boys } \\
\text { Girls }\end{array}$ & $\begin{array}{l}0.305^{\star \star} \\
0.167 \\
0.204\end{array}$ & $\begin{array}{l}0.603^{\star \star} \\
0.551^{\star \star} \\
0.546^{\star \star}\end{array}$ & $\begin{array}{l}-0.321^{\star \star} \\
-0.741^{\star \star} \\
-0.077\end{array}$ & & & & & & & \\
\hline Handgrip & $\begin{array}{l}\text { All } \\
\text { Boys } \\
\text { Girls }\end{array}$ & $\begin{array}{l}0.251^{\star} \\
0.020 \\
0.221\end{array}$ & $\begin{array}{l}0.330^{\star \star} \\
0.211 \\
0.174\end{array}$ & $\begin{array}{l}-0.137 \\
-0.175 \\
-0.122\end{array}$ & $\begin{array}{l}0.372^{\star \star} \\
0.432^{\star \star} \\
0.154\end{array}$ & & & & & & \\
\hline Flamingo & $\begin{array}{l}\text { All } \\
\text { Boys } \\
\text { Girls }\end{array}$ & $\begin{array}{r}0.009 \\
-0.137 \\
-0.075\end{array}$ & $\begin{array}{l}-0.256^{\star \star} \\
-0.432^{\star \star} \\
-0.395^{\star \star}\end{array}$ & $\begin{array}{l}0.349^{\star \star} \\
0.435^{\star \star} \\
0.286^{\star}\end{array}$ & $\begin{array}{l}-0.291^{\star \star} \\
-0.390^{\star \star} \\
-0.442^{\star \star}\end{array}$ & $\begin{array}{r}0.001 \\
-0.181 \\
-0.010\end{array}$ & & & & & \\
\hline Sit-ups & $\begin{array}{l}\text { All } \\
\text { Boys } \\
\text { Girls }\end{array}$ & $\begin{array}{l}0.288^{\star *} \\
0.232 \\
0.210\end{array}$ & $\begin{array}{l}0.576^{\star \star} \\
0.672^{\star \star} \\
0.467^{\star \star}\end{array}$ & $\begin{array}{l}-0.241^{*} \\
-0.469^{\star \star} \\
-0.107\end{array}$ & $\begin{array}{l}0.588^{\star \star} \\
0.471^{\star \star} \\
0.597^{\star \star}\end{array}$ & $\begin{array}{l}0.325^{\star *} \\
0.308^{\star} \\
0.240\end{array}$ & $\begin{array}{l}-0.229^{*} \\
-0.206 \\
-0.380^{\star \star}\end{array}$ & & & & \\
\hline Plate tapping & $\begin{array}{l}\text { All } \\
\text { Boys } \\
\text { Girls }\end{array}$ & $\begin{array}{l}0.052 \\
0.089 \\
0.014\end{array}$ & $\begin{array}{l}0.335^{\star \star} \\
0.369^{\star} \\
0.369^{\star \star}\end{array}$ & $\begin{array}{l}-0.207^{\star} \\
-0.281 \\
-0.144\end{array}$ & $\begin{array}{l}0.286^{\star *} \\
0.372^{\star} \\
0.259\end{array}$ & $\begin{array}{l}0.224^{*} \\
0.304^{*} \\
0.174\end{array}$ & $\begin{array}{l}-0.094 \\
-0.172 \\
-0.036\end{array}$ & $\begin{array}{l}0.274^{\star *} \\
0.242 \\
0.305^{\star}\end{array}$ & & & \\
\hline Sit and reach & $\begin{array}{l}\text { All } \\
\text { Boys } \\
\text { Girls }\end{array}$ & $\begin{array}{r}-0.168 \\
0.220 \\
-0.129\end{array}$ & $\begin{array}{r}-0.023 \\
0.231 \\
0.204\end{array}$ & $\begin{array}{l}-0.206^{\star} \\
-0.477^{\star \star} \\
-0.71\end{array}$ & $\begin{array}{l}0.180 \\
0.408^{\star *} \\
0.394^{\star *}\end{array}$ & $\begin{array}{r}-0.159 \\
0.034 \\
-0.002\end{array}$ & $\begin{array}{l}-0.387^{\star \star} \\
-0.485^{\star \star} \\
-0.218\end{array}$ & $\begin{array}{l}0.149 \\
0.047 \\
0.407^{\star \star}\end{array}$ & $\begin{array}{l}0.120 \\
0.205 \\
0.102\end{array}$ & & \\
\hline$A P_{\text {Math }}(\rho)$ & $\begin{array}{l}\text { All } \\
\text { Boys } \\
\text { Girls }\end{array}$ & $\begin{array}{l}0.576^{* *} \\
-0.018 \\
-0.001\end{array}$ & $\begin{array}{l}-0.128 \\
-0.056 \\
-0.182\end{array}$ & $\begin{array}{c}-0.541^{\star *} \\
-0.249 \\
0.070\end{array}$ & $\begin{array}{r}0.014 \\
0.188 \\
-0.084\end{array}$ & $\begin{array}{l}-0.108 \\
-0.007 \\
-0.156\end{array}$ & $\begin{array}{r}-0.041 \\
-0.213 \\
0.125\end{array}$ & $\begin{array}{l}-0.188 \\
-0.149 \\
-0.189\end{array}$ & $\begin{array}{l}-0.048 \\
-0.035 \\
-0.059\end{array}$ & $\begin{array}{l}0.115 \\
0.214 \\
0.023\end{array}$ & \\
\hline
\end{tabular}

Aerobic power calculated from $20 \mathrm{~m}$ shuttle run $\dot{\mathrm{VO}}_{2 \text { peak }}$ ) following the Leger protocol (Leger and Lambert, 1982) and expressed in $\mathrm{mL}^{\circ} \mathrm{kg}^{-1} \mathrm{~min}{ }^{-1} ; \mathrm{MVAAB}_{\mathrm{OB}}$, physical activity measured directly using SenseWear Armbands in N = 50 sub-sample (21 boys and 29 girls); AP math: academic performance (math grade, corresponding to the final grade awarded at the end of Grade 8); *Significant correlation $p$ < 0.05; * ${ }^{*}$ Significant correlation $\mathrm{p}<0.01$; Italics font represents non-parametric correlations (Spearman rho). 
TABLE 3 | Model summary for the backward stepwise linear regression on the physical fitness change scores from Grade 6 to Grade 9 , exclusively.

\begin{tabular}{|c|c|c|c|c|c|c|c|c|c|}
\hline Model & $\boldsymbol{R}$ & $R^{2}$ & $\begin{array}{c}\text { Adjusted } \\
R^{2}\end{array}$ & $\begin{array}{l}\text { Std. error of } \\
\text { the estimate }\end{array}$ & $\begin{array}{l}\text { Sum of } \\
\text { squares }\end{array}$ & $\begin{array}{l}\text { Degrees of } \\
\text { freedom (df) }\end{array}$ & Mean square & F-statistic & Significance \\
\hline \multicolumn{10}{|l|}{ A } \\
\hline 1 & 0.711 & 0.505 & 0.408 & 0.74268 & 98.020 & 9 & 10.891 & 19.745 & 0.000 \\
\hline 2 & 0.711 & 0.505 & 0.483 & 0.74057 & 98.017 & 8 & 12.252 & 22.340 & 0.000 \\
\hline 3 & 0.711 & 0.505 & 0.485 & 0.73854 & 97.997 & 7 & 14.000 & 25.666 & 0.000 \\
\hline 4 & 0.709 & 0.503 & 0.486 & 0.73827 & 97.522 & 6 & 16.254 & 29.821 & 0.000 \\
\hline 5 & 0.706 & 0.498 & 0.484 & 0.73948 & 96.659 & 5 & 19.353 & 35.353 & 0.000 \\
\hline 6 & 0.703 & 0.494 & 0.483 & 0.74060 & 95.815 & 4 & 23.954 & 43.672 & 0.000 \\
\hline \multicolumn{10}{|l|}{ B } \\
\hline 7 & 0.710 & 0.504 & 0.455 & 0.80156 & 58.816 & 9 & 6.535 & 10.171 & 0.000 \\
\hline 8 & 0.710 & 0.504 & 0.460 & 0.79732 & 58.790 & 8 & 7.349 & 11.560 & 0.000 \\
\hline 9 & 0.709 & 0.503 & 0.465 & 0.79382 & 58.667 & 7 & 8.381 & 13.300 & 0.000 \\
\hline 10 & 0.708 & 0.501 & 0.469 & 0.79097 & 58.456 & 6 & 9.743 & 15.572 & 0.000 \\
\hline 11 & 0.707 & 0.499 & 0.473 & 0.78808 & 58.259 & 5 & 11.652 & 18.761 & 0.000 \\
\hline 12 & 0.704 & 0.495 & 0.474 & 0.78726 & 57.761 & 4 & 14.440 & 23.299 & 0.000 \\
\hline \multicolumn{10}{|l|}{ C } \\
\hline 13 & 0.751 & 0.564 & 0.511 & 0.67202 & 43.283 & 9 & 4.809 & 10.649 & 0.000 \\
\hline 14 & 0.751 & 0.564 & 0.518 & 0.66753 & 43.282 & 8 & 5.410 & 12.142 & 0.000 \\
\hline 15 & 0.751 & 0.564 & 0.524 & 0.66316 & 43.279 & 7 & 6.183 & 14.059 & 0.000 \\
\hline 16 & 0.751 & 0.564 & 0.530 & 0.65893 & 43.270 & 6 & 7.212 & 16.610 & 0.000 \\
\hline 17 & 0.751 & 0.564 & 0.536 & 0.65500 & 43.238 & 5 & 8.648 & 20.156 & 0.000 \\
\hline 18 & 0.748 & 0.559 & 0.537 & 0.65423 & 42.889 & 4 & 10.722 & 25.050 & 0.000 \\
\hline 19 & 0.745 & 0.556 & 0.539 & 0.65269 & 42.622 & 3 & 14.207 & 33.350 & 0.000 \\
\hline 20 & 0.735 & 0.540 & 0.540 & 0.65972 & 41.449 & 2 & 20.724 & 47.617 & 0.000 \\
\hline
\end{tabular}

Data were analyzed for $(A)$ entire sample $(N=184)$, $(B)$ boys $(N=100)$ and $(C)$ girls $(N=84)$, respectively. Final models are bolded for interest.

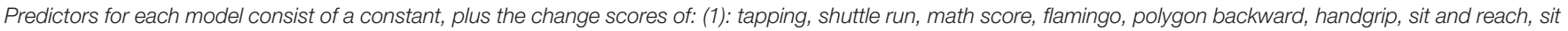

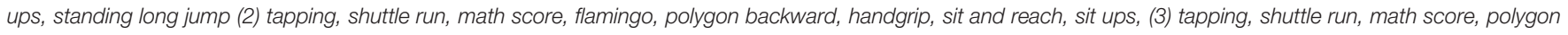

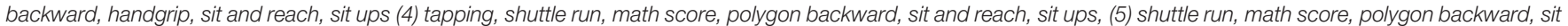

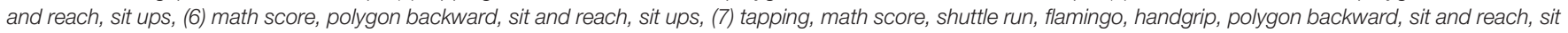

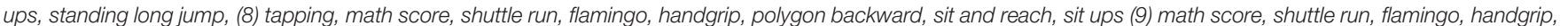

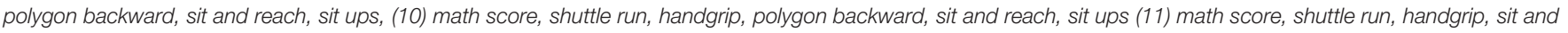

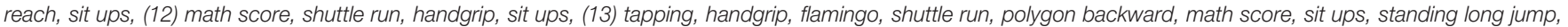
sit and reach, (14) tapping, handgrip, flamingo, shuttle run, polygon backward, math score, sit ups, sit and reach, (15) tapping, handgrip, shuttle run, polygon backward, math score, sit ups, sit and reach, (16) tapping, shuttle run, polygon backward, math score, sit ups, sit and reach, (17) tapping, polygon backward, math score, sit ups,

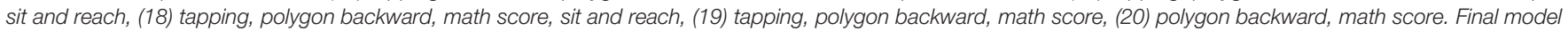
compositions are bolded for interest.

in Grade 9, either for everyone ( $r=0.051, p=0.612)$, for boys $(r=0.018, p=0.908)$ or for girls $(r=0.001, p=0.993)$.

\section{Physical Fitness and Mathematics Academic Performance}

In Grade 6, mathematics grade $\mathrm{AP}_{\text {math }}$ demonstrated a low association with standing long jump $(r=0.247, p=0.010)$; however, only in girls were the results of this test significantly correlated $(r=0.294, p=0.031)$. By Grade 9 , there were no fitness indicators which correlated significantly to math grade for that year. This was true for both sexes, even for peak aerobic power $\left(\dot{\mathrm{V}}_{2 \text { peak }}\right)$, which did not correlate to $\mathrm{AP}_{\text {math }}$ in Grade 9.

\section{Physical Fitness and Activity Predictors of Final Mathematics Grade}

A backward stepwise multiple regression was used to determine which indicators may have shared variance to the youths' final $\mathrm{AP}_{\text {math }}$ scores, awarded at the end of Grade 9, and based on the delta change scores of the fitness measures. Of all the fitness measures, only backward obstacle course, sit and reach, sit-ups, and delta change in $\mathrm{AP}_{\text {math }}$ remained significant to the final model $\left[r^{2}=0.494, F(4,180)=43.67, p<0.0001\right.$, Tables 3, 4]. When this model was run separately by sex, the input variables differed slightly between boys and girls, wherein shuttle run, handgrip, sit-ups and $\triangle \mathrm{AP}_{\text {math }}$ contributed to the final model for boys $\left[r^{2}=0.495, F(4,95)=23.299, p<0.0001\right]$, and only backward obstacle course and $\triangle \mathrm{AP}_{\text {math }}$ contributed to the girls' model $\left[r^{2}=0.540, F(2,81)=47.7, p<0.0001\right]$.

Determining the (possible) effect of MVPA on final $\mathrm{AP}_{\text {math }}$ scores required a second analysis since $\mathrm{MVPA}_{\mathrm{OB}}$ were obtained in a sub-sample of youth and not the entire cohort. In this scenario, MVPA $_{\mathrm{OB}}$ attained its lowest $p$-value of 0.188 , a standardized beta of -0.122 , and was thus removed from the model on step 7 of 9 (Tables 5, 6). Likewise, when data were separated by sex, MVPA $_{\mathrm{OB}}$ it did not reach significance for either analysis, being removed from the model at step $3(p=0.698)$ and step 7 ( $p=0.315$ ) for boys and girls, respectively, and therefore, only combined data are detailed in subsequent tables. 


\section{DISCUSSION}

The results of this study found that longitudinal fitness changes in Slovenian youth were related to changes in objectively assessed physical activity $\left(\mathrm{MVPA}_{\mathrm{OB}}\right)$, including small associations between standing long jump and MVPA ${ }_{\mathrm{OB}}$.
Mathematics score decreased within this 3-year timespan for both boys and girls. The final mathematics grade at the end of primary school was associated with changes in whole-body coordination (backward obstacle course), flexibility (sit and reach) and muscular endurance (sit-ups) when boys' and girls' data were combined. When separated, fitness indicators differed

TABLE 4 | Coefficients data for the first and final model for $(A)$ entire sample $(N=184)$, (B) boys $(N=100)$, and $(C)$ girls $(N=84)$, respectively.

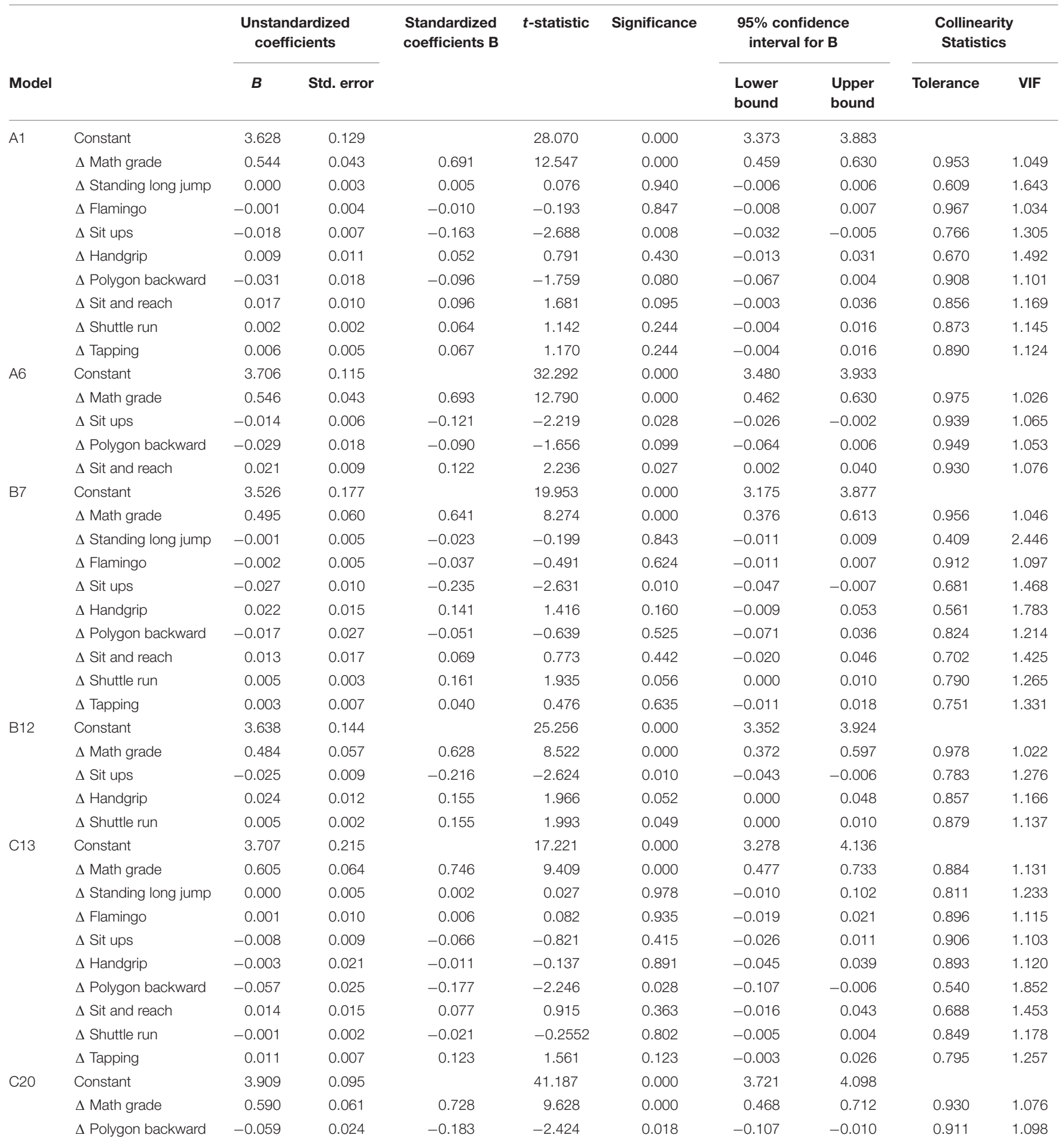


TABLE 5 | Model summary for the backward stepwise linear regression on the physical fitness change scores from Grade 6 to Grade 9 , with the inclusion of MVPAOB.

\begin{tabular}{|c|c|c|c|c|c|c|c|c|c|}
\hline Model & $\boldsymbol{R}$ & $R^{2}$ & Adjusted $R^{2}$ & $\begin{array}{c}\text { Std. error of the } \\
\text { estimate }\end{array}$ & $\begin{array}{l}\text { Sum of } \\
\text { squares }\end{array}$ & $\begin{array}{l}\text { Degrees of } \\
\text { freedom (df) }\end{array}$ & Mean square & $F$-statistic & Significance \\
\hline 1 & 0.851 & 0.724 & 0.625 & 0.62011 & 28.207 & 10 & 2.821 & 7.335 & 0.000 \\
\hline 2 & 0.851 & 0.724 & 0.638 & 0.60954 & 28.200 & 9 & 3.133 & 8.433 & 0.000 \\
\hline 3 & 0.849 & 0.721 & 0.646 & 0.60258 & 28.081 & 8 & 3.510 & 9.667 & 0.000 \\
\hline 4 & 0.849 & 0.716 & 0.652 & 0.59753 & 27.906 & 7 & 3.978 & 11.166 & 0.000 \\
\hline 5 & 0.843 & 0.711 & 0.656 & 0.59379 & 27.692 & 6 & 4.615 & 13.090 & 0.000 \\
\hline 6 & 0.837 & 0.701 & 0.655 & 0.59453 & 27.310 & 5 & 5.462 & 15.453 & 0.000 \\
\hline 7 & 0.827 & 0.684 & 0.647 & 0.60153 & 26.672 & 4 & 6.668 & 18.428 & 0.000 \\
\hline 8 & 0.815 & 0.664 & 0.635 & 0.61196 & 25.867 & 3 & 8.622 & 23.024 & 0.000 \\
\hline 9 & 0.799 & 0.638 & 0.618 & 0.61258 & 24.873 & 2 & 12.436 & 31.749 & 0.000 \\
\hline
\end{tabular}

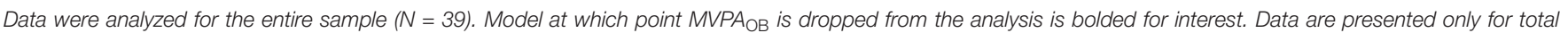
data set, not stratified by sex.

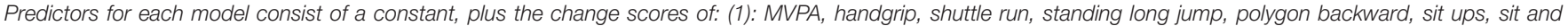

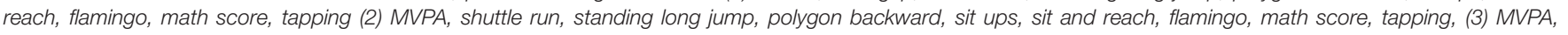
shuttle run, standing long jump, polygon backward, sit ups, sit and reach, flamingo, math score, (4) MVPA, standing long jump, polygon backward, sit ups, sit and reach,

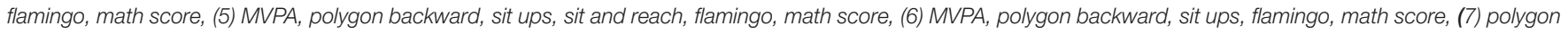
backward, sit ups, flamingo, math score, (8) polygon backward, sit ups, math score, (9) sit ups, math score.

TABLE 6 | Coefficients data for the first and final model with the inclusion of MVPAOB.

\begin{tabular}{|c|c|c|c|c|c|c|c|c|c|c|}
\hline \multirow[b]{2}{*}{ Model } & & \multicolumn{2}{|c|}{$\begin{array}{l}\text { Unstandardized } \\
\text { coefficients }\end{array}$} & \multirow[t]{2}{*}{$\begin{array}{l}\text { Standardized } \\
\text { coefficients } B\end{array}$} & \multirow[t]{2}{*}{$t$-statistic } & \multirow[t]{2}{*}{ Significance } & \multicolumn{2}{|c|}{$\begin{array}{c}95 \% \text { confidence } \\
\text { interval for B }\end{array}$} & \multicolumn{2}{|c|}{$\begin{array}{l}\text { Collinearity } \\
\text { statistics }\end{array}$} \\
\hline & & $B$ & Std. error & & & & $\begin{array}{l}\text { Lower } \\
\text { bound }\end{array}$ & $\begin{array}{l}\text { Upper } \\
\text { bound }\end{array}$ & Tolerance & VIF \\
\hline \multirow[t]{10}{*}{1} & Constant & 5.029 & 0.572 & & 8.788 & 0.000 & 3.857 & 6.201 & & \\
\hline & $\Delta$ Math grade & 0.650 & 0.092 & 0.786 & 7.087 & 0.000 & 0.462 & 0.837 & 0.803 & 1.245 \\
\hline & $\Delta$ Standing long jump & -0.004 & 0.006 & -0.067 & -0.644 & 0.525 & -0.016 & 0.008 & 0.916 & 1.092 \\
\hline & $\Delta$ Flamingo & 0.025 & 0.017 & 0.161 & 1.459 & 0.156 & -0.010 & 0.059 & 0.814 & 1.229 \\
\hline & $\Delta$ Sit ups & -0.025 & 0.014 & -0.195 & -1.897 & 0.081 & -0.053 & 0.003 & 0.850 & 1.176 \\
\hline & $\Delta$ Handgrip & 0.004 & 0.026 & 0.016 & 0.140 & 0.890 & -0.049 & 0.056 & 0.782 & 1.279 \\
\hline & $\Delta$ Polygon backward & -0.052 & 0.032 & -0.179 & -1.640 & 0.112 & -0.117 & 0.013 & 0.825 & 1.213 \\
\hline & $\Delta$ Sit and reach & -0.021 & 0.021 & -0.110 & -0.997 & 0.327 & -0.065 & 0.023 & 0.812 & 1.231 \\
\hline & $\Delta$ Shuttle run & 0.003 & 0.004 & 0.096 & 0.819 & 0.420 & -0.004 & 0.010 & 0.715 & 1.399 \\
\hline & $\Delta$ Tapping & 0.006 & 0.011 & 0.066 & 0.571 & 0.572 & -0.017 & 0.030 & 0.746 & 1.340 \\
\hline \multirow[t]{3}{*}{9} & Constant & 4.071 & 0.137 & & 29.730 & 0.000 & 3.793 & 4.349 & & \\
\hline & $\Delta$ Math grade & 0.621 & 0.084 & 0.751 & 7.415 & 0.000 & 0.451 & 0.791 & 0.980 & 1.021 \\
\hline & $\Delta$ Sit ups & -0.024 & 0.013 & -0.185 & -1.828 & 0.076 & -0.050 & 0.003 & 0.980 & 1.021 \\
\hline
\end{tabular}

Data were analyzed for the entire sample $(N=39)$. Data are presented only for total data set, not stratified by sex.

such that for boys, aerobic power (shuttle run), upper body strength (handgrip), and muscular endurance (sit-ups) shared variance with final math score whereas whole-body coordination backward obstacle course was the more relevant fitness indicator to girls' final math score.

\section{Longitudinal Changes in Physical Activity and Fitness}

Physical activity levels in children tend to decrease with age, and a recent meta-analysis has demonstrated that between the ages 11 and 14 (which correspond to grades 6 and 9 in Slovenia), MVPA declines similarly amongst boys and girls at a rate of around $4.3 \%$ and $4.5 \%$ per year, respectively (Farooq et al., 2020). Analysis from the current investigation, and trends from the ongoing SLOfit surveillance (Jurak et al., 2020), show larger declining trends in Slovenian boys and girls, and greater differences between them. For example, girls experience a $\sim 15.2 \%$ annual decline, thus experiencing 2.6-times larger annual declines in MVPA than boys, who see declines of $\sim 5.7 \%$ annually. Although the declines in MVPA observed between Grades 6 and 9 can be possibly linked to greater school-related workloads (e.g., compulsory annual instruction time increases from 700 to $892.5 \mathrm{~h}$, respectively), and the reduced hours of compulsory physical education after Grade 6 from 105 to $70 \mathrm{~h}$ per year, this workload increase and school-related PA is equal for boys and girls, and cannot in and of itself explain the growing sex-effect difference observed in MVPA in the present study.

Similarly, there are growing disparities between boys and girls when observing changes in aerobic power, measured via the shuttle run. In boys, this indicator of cardiorespiratory 
fitness increased from 45 to $53 \mathrm{~mL} \cdot \mathrm{kg}^{-1} \cdot \mathrm{min}^{-1}$ between Grade 6 and 9, whereas for girls, their values decreased from 46 to $41 \mathrm{~mL} \cdot \mathrm{kg}^{-1} \cdot \mathrm{min}^{-1}$. In the Muscatine study, both boys and girls experienced a decline of $\mathrm{VO}_{2 \text { peak }}$ in this age period (Janz et al., 2000), although in that study, the level of oxygen consumption was much lower than our recorded level, and therefore any changes observed in MVPA for our cohort may have had greater relative effects compared to a less-fit group of children at baseline.

Coherent to the trends in MVPA, boys in our study did not experience a declining trend in any other physical fitness test (other than the flamingo balance test) whilst girls experienced improvement in all physical fitness indicators, despite recorded declines in MVPA. Indeed, girls even exceeded the boys' performance in terms of the flamingo balance test and sit-andreach scores, whereas boys achieved higher results in standing long jump and handgrip test. These results suggest that girls may achieve higher levels of neuromuscular fitness by age 14 compared to their male counterparts, which could be linked to their earlier timing of puberty/maturation, and consequently more developed central nervous system at this age (Hoyt et al., 2020). Unfortunately, direct assessment of maturation was outside of the scope of this study, so any improvements in these measures must be considered when interpreting the data. In flexibility, girls also tended to achieve higher results throughout childhood and adolescence. By age 14, boys tended to achieve higher levels of muscular fitness which is linked to increasing muscle mass and consequent muscle power, likely because of higher testosterone levels as puberty continues to progress (Handelsman, 2017).

\section{Mathematics Academic Performance in Primary School}

In the present study, mathematics grades decreased between Grade 6 and Grade 9 from $\sim 4.2$ to $\sim 3.5$. This magnitude of decrease is not surprising, given that Grade 5 is the last year a generalized education teacher is the one who administers the mathematics curricula. By Grade 6, the youth are taught by a specialist mathematics teacher, and both the volume of work and difficulty level are increased dramatically compared to early primary schooling periods. Population data from national assessments indicate that a typical decrease in math grade does occur, with the average score at the end of Grade 9 being 3.3 (Perger, 2021). Therefore, our results are in-line with national standards for this academic performance metric.

\section{Physical Fitness and Academic Performance in Children and Youth}

Of all the fitness indicators, positive AP is often specifically associated with higher aerobic function (Sardinha et al., 2016). For example, on a sample of boys from Spain (Torrijos-Niño et al., 2014), the authors found that AP was positively related to aerobic fitness, and that obese boys had lower AP scores compared to overweight or normal weight boys. Unsurprisingly, their data suggest that 'academically superior' PF in children and youth are likely the result of lifestyle patterns, including discipline in school-related work and habitual daily PA, a pattern of evidence also reflected in other studies (Donnelly et al.,
2016). Currently, although shuttle run (and by extension, aerobic power) epidemiological literature for children and youth is very well-documented (Tomkinson et al., 2017; Lang et al., 2019), there are few examples where negative fitness and obesity trends have been reversed, especially over the past 20 years. To this point, the Republic of Slovenia has demonstrated a net increase in CRF between 1993 and 2013 in both boys and girls, despite initial decreases observed from 1993 to 2003 (Morrison et al., 2021). Indeed, Slovenian schoolchildren universally meet or exceed international standards for CRF cut-off values for minimizing future cardiovascular health risk. Whether this higher universal fitness standard affects AP in these children and youth are yet to be determined, but the present study did not relate shuttle run aerobic power to mathematics grade per se for either sex within a given grade. Instead, the 3year longitudinal changes observed in aerobic power were a predictor for final math score for boys (but not for girls), indicating both a possible reduction in statistical power when the regression was split by sex, and also the interaction effect present in that metric, where boys saw a net increase in relative function between Grade 6 to Grade 9 (from $46 \pm 26$ to $\left.64 \pm 25 \mathrm{~mL}^{-1} \cdot \mathrm{kg}^{-1} \cdot \mathrm{min}^{-1}\right)$, whereas girls demonstrated the reverse trend (from $46 \pm 27$ to $42 \pm 20 \mathrm{~mL}^{-1} \cdot \mathrm{kg}^{-1} \cdot \mathrm{min}^{-1}$ ). It would be interesting to compare these longitudinal changes in PF and AP in Slovenian schoolchildren to others across Europe, the OECD, and internationally, to determine to what degree increasing PF directly improves AP in a dose-response manner, especially for children who may be starting from a more sedentary and lower aerobic fitness standard.

Previous meta-analyses have found that, in addition to cardiorespiratory fitness, speed-agility, motor coordination, and perceptual motor skills are each highly associated with AP (RuizAriza et al., 2017), but evidence for strength and flexibility remained unclear. Certainly, some authors consider that motor competence is 'indissoluble' from cognitive competence, a model in which cognitive skills arise from motor action (AVILÉS et al., 2014), with motor coordination being one of the most important factors to the evolutionary development of children (Fernandes et al., 2016; Ruiz-Pérez et al., 2016). The present study found a small proportion of the variance in final math grade could be attributed to physical fitness components encompassing motor coordination (backward obstacle course), flexibility (sitand-reach) and strength (sit-ups). These results support the theory of association between body-kinaesthetic intelligence and coordinative performance, which is further reinforced by neural connections existing across structural/muscular factors (Diamond and Lee, 2011). Sit-ups may have contributed to the final model because it is an isokinetic endurance test which considers not only a child's physical abilities, but also psychological characteristics like persistence, a necessary component to any learning process. Our results are consistent with a similar analysis conducted on a sample of boys from Spain (Torrijos-Niño et al., 2014), and a study where the odds ratio for effects of $\mathrm{PF}$ improvement on $\mathrm{AP}$, and mathematics specifically, were calculated for highly fit and unfit children (Sardinha et al., 2016).

In our analysis, we were not able to prove the interrelatedness of objectively assessed PA on AP, which proposes that PF 
indicators may be more insightful than PA alone when studying this phenomenon. Firstly, the information on MVPA alone cannot indicate whether a person is fit or not. This is related to the second limitation of reliability of MVPA assessment, namely, that despite the established recommendations that MVPA should be recorded for several consecutive days, this does not guarantee that actual PA is representative for long-term habitual PA during the observed days. In this regard, PF could serve as a more reliable indicator of habitual PA, since the level of PF is the direct result of long-term habitual PA as an important determinant of phenotype (Sallis et al., 1993a,b). Indeed, due to interpersonal differences in body metabolism, individuals require different frequency, intensity, and duration of PA to obtain or preserve certain levels of PF, or related health benefits (Oja, 2001). Thirdly, $\mathrm{PF}$ seems to be more direct indicator of physiological functioning of human organism which determines also cognitive functioning than PA, and also the effect of PA is itself moderated by PF (Lopes and Rodrigues, 2021).

\section{Espousing Systematic Physical Fitness Monitoring in the Young}

There is clear evidence that monitoring PF in children can be critical to maintaining this key health indicator (Ortega et al., 2008). Higher levels of PF are associated with greater PA (Wang, 2019), including better socialization (Li and Li, 2018) and academic performance (Sember et al., 2020b) and indeed, fitness testing is much more than just 'one more school assessment' since it helps increase awareness of how one's body moves through space. How fit a child is now can relate to how fit and active they become as adults (Kvaavik et al., 2009). Those who have high 'physical literacy' (i.e., are attuned to how their body works and what it needs to function properly), are better able to foster life-long physical activity habits (Whitehead, 2010). Importantly, skill-based fitness developments are legitimate manifestations of physical literacy development since physical literacy is a multidimensional and interactive construct comprising of the physical, behavior, cognitive and affective domain (Whitehead, 2010). Thus, PF is a critical component to physical literacy overall. Additionally, monitoring fitness in schools is an effective policy making tool since there are well-educated professionals (teachers and others) who can ensure effective and accurate student fitness measurements in a timely and accurate fashion (e.g., SLOfit) (Jurak et al., 2019). Monitoring fitness as children mature offers education and public health decision-makers opportunity to respond quickly and effectively to changes in child PF trends, a critical pillar of any public health strategy since globally, children have become more inactive and overweight (Aubert et al., 2018).

It is important to note that fitness testing per se should never be used to grade the student. The assessment of PF is conducted to provide professionals with accurate measures of educational achievement, the health and functional status of the children, and can act as an operational starting point for setting individual goals and tailoring curricula to individual needs. It must be clearly communicated as such to the children as well so that they treat these examinations in context. Timely testing allows for professionals to gain an understanding of the child's educational development and health status to make informed decisions regarding education or further treatment (Lloyd et al., 2010). This is an appropriate way to run effective physical education classes, where students understand the rationale for standardized testing.

Data from the current investigation were possible only because there is a concerted, ongoing effort to chart the fitness changes of the Slovenian schoolchildren population to inform policy making decisions and affect positive public health decisions. Data from fitness databases in Slovenia have contributed to finding that there was a reversal in the negative trends of child aerobic fitness (Morrison et al., 2021), charting pediatric rates of obesity (Potoènik et al., 2020), and identifying how the COVID-19 pandemic has affected child fitness overall (Jurak et al., 2021). By utilizing near real-time health status changes in youth, this study demonstrates how implementing a robust fitness surveillance monitoring program at a population level can detect meaningful changes in health status, even when societal and global perturbations dramatically alter civic life (e.g., conflicts, pandemics, and recessions). Often during times of social transition, decreases in opportunities to engage in quality physical activity may diminish one's physical fitness, especially for vulnerable populations, like the young. Data from the current investigation underscore that a variety of physical fitness indicators are related to changes in academic performance, especially for mathematics grade, and these fitness markers are heterogeneous between boys and girls. Only by continuously monitoring fitness can one make changes to regional, national, and international policies which can then have lasting trickledown effects on longstanding societal health issues like obesity, chronic hypertension, stroke, as well as effects like cognition, academic performance, fitness, and mental health.

\section{CONCLUSION}

Girls and boys in Slovenia are becoming less physically active when progressing from Grade 6 to Grade 9. Decreases are also evident for AP in mathematics in both girls and boys during this timeframe, likely reflecting structural issues present within the Slovenian primary education system (i.e., students aged $\sim 6-14$ years), rather than other external or physical fitness factors alone. In contrast to prevailing existing evidence which link AP with cardiorespiratory fitness lower math grades in our study are also moderately associated with indicators of muscular and neuro-muscular fitness, including changes in whole-body coordination, flexibility, and muscular endurance for the entire sample. Aerobic power, upper body strength, and muscular endurance share more common variance to final math grade in boys, whereas whole-body coordination was the more relevant index in girls; this finding suggests that future research exploring the relationship of AP and PF should not be limited to cardiorespiratory PF and should also encompass muscular and neuro-muscular components of PF. Our study suggests that PF can serve as more useful indicator for assessment of AP risks than PA, but also that the current data indicates the association between PF and PA declines with age. The reason of this decline remains unclear but directs researchers that toward adolescence, factors other than PF may be more detrimental to AP in general, and mathematics grade specifically. 


\section{DATA AVAILABILITY STATEMENT}

The raw data supporting the conclusions of this article will be made available by the authors, without undue reservation, to any qualified researcher.

\section{ETHICS STATEMENT}

The studies involving human participants were reviewed and approved by the Slovenian National Medical Ethics Committee (ID:138/05/13), following the Declaration of Helsinki. Written informed consent was obtained from the minor's legal guardian/next of kin (and assent from the child) to participate in the study.

\section{AUTHOR CONTRIBUTIONS}

VS: investigation, conceptualization, formal analysis, visualization, writing-original draft, writing-review and editing, and writing-approval of final manuscript. GJ: project

\section{REFERENCES}

Aubert, S., Barnes, J., Abdeta, C., Abi Nader, P., Adeniyi, A., Aguilar-Farias, N., et al. (2018). Global matrix 3.0 physical activity report card grades for children and youth: results and analysis from 49 countries. J. Phys. Activ. Health 15, S251-S273. doi: 10.1123/jpah.2018-0472

Avilés, C., Ruiz-Pérez, L. M., Navia, J. A., Rioja, N., and Sanz, D. (2014). La pericia perceptivo-motriz y cognición en el deporte: del enfoque ecológico y dinámico a la enacción. Ann. Psychol. 30, 725-737.

Beck, M. M., Lind, R. R., Geertsen, S. S., Ritz, C., Lundbye-Jensen, J., and Wienecke, J. (2016). Motor-enriched learning activities can improve mathematical performance in preadolescent children. Front. Hum. Neurosci. 10:645. doi: 10. 3389/fnhum.2016.00645

Biddle, S. J. H., and Asare, M. (2011). Physical activity and mental health in children and adolescents: a review of reviews. Br. J. Sports Med. 45, 886-895. doi: 10.1136/bjsports-2011-090185

Cadenas-Sanchez, C., Migueles, J. H., Erickson, K. I., Esteban-Cornejo, I., Catena, A., and Ortega, F. B. (2020). Do fitter kids have bigger brains? Scand. J. Med. Sci. Sports 30, 2498-2502. doi: 10.1111/sms.13824

Cain, K. L., Sallis, J. F., Conway, T. L., Van Dyck, D., and Calhoon, L. (2013). Using accelerometers in youth physical activity studies: a review of methods. J. Phys. Activ. Health 10, 437-450. doi: 10.1123/jpah.10.3.437

Calabró, M. A., Welk, G. J., and Eisenmann, J. C. (2009). Validation of the SenseWear Pro Armband algorithms in children. Med. Sci. Sports Exerc. 41, 1714-1720. doi: 10.1249/MSS.0b013e3181a071cf

Carlson, S. A., Fulton, J. E., Lee, S. M., Maynard, L. M., Brown, D. R. III, Dietz, H. W., et al. (2008). Physical education and academic achievement in elementary school?: data from the early childhood longitudinal study. Am. J. Public Health 98, 721-727. doi: 10.2105/AJPH.2007.117176

Caspersen, C. J., Powell, K. E., and Christenson, G. M. (1985). Physical activity, exercise, and physical fitness: definitions and distinctions for health-related research. Public Health Rep. 100, 126-131.

Castelli, D. M., Hillman, C. H., Buck, S. M., and Erwin, H. E. (2007). Physical fitness and academic achievement in third-and fifth-grade students. J. Sport Exerc. Psychol. 29, 239-252. doi: 10.1123/jsep.29.2.239

Catellier, D. J., Hannan, P. J., Murray, D. M., Addy, C. L., Conway, T. L., Yang, S., et al. (2005). Imputation of missing data when measuring physical activity by accelerometry. Med. Sci. Sports Exerc. 37(11 Suppl.):S555. doi: 10.1249/01.mss. $0000185651.59486 .4 \mathrm{e}$ administration, investigation, conceptualization, resources, writing-review and editing, and writing-approval of final manuscript. GS: project administration, investigation, conceptualization, resources, writing - review and editing, and writing-approval of final manuscript. SM: conceptualization, formal analysis, visualization, writing-original draft, writingreview and editing, and writing - approval of final manuscript. All authors contributed to the article and approved the submitted version.

\section{FUNDING}

Partial, non-specific funding was received by the Slovenian National Research Agency (ARRS), grant number P5-0142. The study received invaluable support with sport equipment donated to the participating schools from the Slovenian Olympic Committee and Elan Inventa, a Slovenian sporting manufacturer company and supplier. The companies had no knowledge or influence of the present study regarding data collection, analysis, or publication of the work transpiring from their donations.

Chaddock, L., Erickson, K. I., Prakash, R. S., VanPatter, M., Voss, M. W., Pontifex, M. B., et al. (2010). Basal ganglia volume is associated with aerobic fitness in preadolescent children. Dev. Neurosci. 32, 249-256. doi: 10.1159/000316648

Chaddock, L., Pontifex, M. B., Hillman, C. H., and Kramer, A. F. (2011). A review of the relation of aerobic fitness and physical activity to brain structure and function in children. J. Intern. Neuropsychol. Soc. 17, 975-985. doi: 10.1017/ S1355617711000567

Chaddock-Heyman, L., Erickson, K. I., Holtrop, J. L., Voss, M. W., Pontifex, M. B., Raine, L. B., et al. (2014). Aerobic fitness is associated with greater white matter integrity in children. Front. Hum. Neurosci. 8:584. doi: 10.3389/fnhum.2014. 00584

Chaddock-Heyman, L., Erickson, K. I., Voss, M., Knecht, A., Pontifex, M. B., Castelli, D., et al. (2013). The effects of physical activity on functional MRI activation associated with cognitive control in children: a randomized controlled intervention. Front. Hum. Neurosci. 7:72. doi: 10.3389/fnhum.2013. 00072

Coe, D. P., Pivarnik, J. M., Womack, C. J., Reeves, M. J., and Malina, R. M. (2006). Effect of physical education and activity levels on academic achievement in children. Med. Sci. Sports Exerc. 38, 1515-1519. doi: 10.1249/01.mss. $0000227537.13175 .1 \mathrm{~b}$

Daly-Smith, A. J., Zwolinsky, S., McKenna, J., Tomporowski, P. D., Defeyter, M. A., and Manley, A. (2018). Systematic review of acute physically active learning and classroom movement breaks on children's physical activity, cognition, academic performance and classroom behaviour: understanding critical design features. BMJ Open Sport Exerc. Med. 4, 1-16. doi: 10.1136/bmjsem-2018-000341

Diamond, A., and Lee, K. (2011). Interventions shown to aid executive function development in children 4 to 12 years old. Science 333, 959-964. doi: 10.1126/ science. 1204529

Donnelly, J. E., Hillman, C. H., Castelli, D., Etnier, J. L., Lee, S., Tomporowski, P., et al. (2016). Physical activity, fitness, cognitive function, and academic achievement in children: a systematic review. Med. Sci. Sports Exerc. 48:1197.

Dowd, K. P., Szeklicki, R., Minetto, M. A., Murphy, M. H., Polito, A., Ghigo, E., et al. (2018). A systematic literature review of reviews on techniques for physical activity measurement in adults: a DEDIPAC study. Intern. J. Behav. Nutr. Phys. Activ. 15:15. doi: 10.1186/s12966-017-0636-2

Duffey, K., Barbosa, A., Whiting, S., Mendes, R., Aguirre, I. Y., Tcymbal, A., et al. (2021). Barriers and facilitators of physical activity participation in adolescent girls: a systematic review of systematic reviews. Front. Public Health 9:743935. doi: 10.3389/fpubh.2021.743935 
Esteban-Cornejo, I., Mora-Gonzalez, J., Cadenas-Sanchez, C., ContrerasRodriguez, O., Verdejo-Román, J., Henriksson, P., et al. (2019). Fitness, cortical thickness and surface area in overweight/obese children: the mediating role of body composition and relationship with intelligence. Neuroimage 186, 771-781. doi: 10.1016/j.neuroimage.2018.11.047

Eveland-sayers, B. M., Farley, R. S., Fuller, D. K., Morgan, D. W., and Caputo, J. L. (2009). Physical fitness and academic achievement in elementary school children. J. Phys. Activ. Health 6, 99-104. doi: 10.1123/jpah.6.1.99

Farooq, A., Martin, A., Janssen, X., Wilson, M. G., Gibson, A. M., Hughes, A., et al. (2020). Longitudinal changes in moderate-to-vigorous-intensity physical activity in children and adolescents: a systematic review and meta-analysis. Obes. Rev. 21:e12953. doi: 10.1111/obr.12953

Fernhall, B., and Agiovlasitis, S. (2008). Arterial function in youth: window into cardiovascular risk. J. Appl. Physiol. 105, 325-333. doi: 10.1152/japplphysiol. 00001.2008

Fernandes, V. R., Ribeiro, M. L. S., Melo, T., de Tarso Maciel-Pinheiro, P., Guimarães, T. T., Araújo, N. B., et al. (2016). Motor coordination correlates with academic achievement and cognitive function in children. Front. Psychol. 7:318. doi: 10.3389/fpsyg.2016.00318

Flere, S., Klanjšek, R., Musil, B., Tavèar Krajnc, M., and Kirbiš, A. (2009). Kdo je Uspešen $v$ Slovenski Šoli. Znanstveno Poroèilo [Who Is Successful in the Slovene School. Ljubljana: Peda-goski Institute.

Fletcher, G. F., Balady, G., Blair, S. N., Blumenthal, J., Caspersen, C., Chaitman, B., et al. (1996). Statement on exercise: benefits and recommendations for physical activity programs for all Americans: a statement for health professionals by the Committee on Exercise and Cardiac Rehabilitation of the Council on Clinical Cardiology. Am. Heart Assoc. Circ. 94, 857-862.

Fredericks, C. R., Kokot, S. J., and Krog, S. (2006). Using a developmental movement programme to enhance academic skills in grade 1 learners. South Afr. J. Res. Sport Phys. Educ. Recreat. 28, 29-42.

García-Hermoso, A., Ramírez-Vélez, R., Lubans, D. R., and Izquierdo, M. (2021). Effects of physical education interventions on cognition and academic performance outcomes in children and adolescents: a systematic review and meta-analysis. Br. J. Sports Med. 55, 1224-1232. doi: 10.1136/bjsports-2021104112

Gunter, K. B., Almstedt, H. C., and Janz, K. F. (2012). Physical activity in childhood may be the key to optimizing lifespan skeletal health. Exerc. Sport Sci. Rev. 40:13. doi: 10.1097/JES.0b013e318236e5ee

Handelsman, D. J. (2017). Sex differences in athletic performance emerge coinciding with the onset of male puberty. Clin. Endocrinol. 87, 68-72. doi: $10.1111 /$ cen. 13350

Hoyt, L. T., Niu, L., Pachucki, M. C., and Chaku, N. (2020). Timing of puberty in boys and girls: implications for population health. SSM Popul. Health 10:100549.

Janssen, I., and LeBlanc, A. G. (2010). Systematic review of the health benefits of physical activity and fitness in school-aged children and youth. Intern. J. Behav. Nutr. Phys. Activ. 7:40. doi: 10.1186/1479-5868-7-40

Janz, K. F., Dawson, J. D., and Mahoney, L. T. (2000). Tracking physical fitness and physical activity from childhood to adolescence: the Muscatine study. Med. Sci. Sports Exerc. 32, 1250-1257.

Jurak, G., Kovaè, M., and Starc, G. (2013). The ACDSi 2013-the analysis of children's development in Slovenia 2013: study protocol. Anthropol. Noteb. 19, 123-143.

Jurak, G., Leskošek, B., Kovaè, M., Sorić, M., Kramaršiè, J., Sember, V., et al. (2020). SLOfit surveillance system of somatic and motor development of children and adolescents: upgrading the Slovenian sports educational chart. Auc. Kinanthropol. 56, 28-40.

Jurak, G., Morrison, S. A., Kovaè, M., Leskošek, B., Sember, V., Strel, J., et al. (2021). A COVID-19 crisis in child physical fitness: Creating a barometric tool of public health engagement for the Republic of Slovenia. Front. Public Health 9:644235. doi: 10.3389/fpubh.2021.644235

Jurak, G., Sember, V., and Starc, G. (2019). 30 Years of SLOfit?: its legacy and perspective. Turk. J. Sports Med. 54, 23-27. doi: 10.5152/tjsm.2019.148

Kvaavik, E., Klepp, K.-I., Tell, G. S., Meyer, H. E., and Batty, G. D. (2009). Physical fitness and physical activity at age 13 years as predictors of cardiovascular disease risk factors at ages $15,25,33$, and 40 years: extended follow-up of the Oslo youth study. Pediatrics 123, e80-e86. doi: 10.1542/peds.2008-1118
Lang, J. J., Tremblay, M. S., Ortega, F. B., Ruiz, J. R., and Tomkinson, G. R. (2019). Review of criterion-referenced standards for cardiorespiratory fitness: what percentage of 1142026 international children and youth are apparently healthy? Br. J. Sports Med. 53, 953-958.

Leger, L. A., and Lambert, J. (1982). A maximal multistage 20-m shuttle run test to predict VO2 max. Eur. J. Appl. Physiol. Occup. Physiol. 49, 1-12. doi: $10.1007 / \mathrm{BF} 00428958$

Li, J., and Li, Y. (2018). "Research on community games space planning and design strategies for helping children's socialization," in Proceedings of the 2nd International Conference on Culture, Education and Economic Development of Modern Society (ICCESE 2018), Amsterdam.

Lloyd, M., Colley, R. C., and Tremblay, M. S. (2010). Advancing the debate on 'fitness testing'for children: Perhaps we're riding the wrong animal. Pediatr. Exerc. Sci. 22, 176-182. doi: 10.1123/pes.22.2.176

Lopes, V. P., and Rodrigues, L. P. (2021). The role of physical fitness on the relationship between motor competence and physical activity: mediator or moderator? J. Mot. Learn. Dev. 1, 1-14.

Mahar, M. T., Guerieri, A. M., Hanna, M. S., and Kemble, C. D. (2011). Estimation of aerobic fitness from 20-m multistage shuttle run test performance. Am. J. Prevent. Med. 41, S117-S123. doi: 10.1016/j.amepre.2011.07.008

Monyeki, M. A., Moss, S. J., Kemper, H. C. G., and Twisk, J. W. R. (2018). Self-reported physical activity is not a valid method for measuring physical activity in 15-year-old south african boys and girls. Children 5:71. doi: 10.3390/ children5060071

Morrison, S. A., Sember, V., Leskošek, B., Kovaè, M., Jurak, G., and Starc, G. (2021). Assessment of secular trends and health risk in pediatric cardiorespiratory fitness from the Republic of Slovenia. Front. Physiol. 12:644781. doi: 10.3389/ fphys.2021.644781

Oja, P. (2001). Dose response between total volume of physical activity and health and fitness. Med. Sci. Sports Exerc. 33(6 Suppl.), S428-S437. doi: 10.1097/ 00005768-200106001-00011

Ortega, F. B., Ruiz, J. R., Castillo, M. J., and Sjöström, M. (2008). Physical fitness in childhood and adolescence: a powerful marker of health. Intern. J. Obes. 32, 1-11. doi: 10.1038/sj.ijo.0803774

Perger, O. (2021). Primerjava Matematiènih Dosežkov Uèencev s Primanjkljaji pri Uèenju Matematike na Nacionalnem Preverjanju Znanja v 6. in 9. Razredu. Ljubljana: Univerza v Ljubljani.

Phillips, D., Hannon, J. C., and Castelli, D. M. (2015). Effects of vigorous intensity physical activity on mathematics test performance. J. Teach. Phys. Educ. 34, 346-362.

Potoènik, ŽL., Jurak, G., and Starc, G. (2020). Secular trends of physical fitness in twenty-five birth cohorts of Slovenian children: a population-based study. Front. Public Health 8:561273. doi: 10.3389/fpubh.2020.561273

Rauner, R. R., Walters, R. W., Avery, M., and Wanser, T. J. (2013). Evidence that aerobic fitness is more salient than weight status in predicting standardized math and reading outcomes in fourth-through eighth-grade students. J. Pediatr. 163, 344-348. doi: 10.1016/j.jpeds.2013.01.006

Reid, R. E. R., Fillon, A., Thivel, D., Henderson, M., Barnett, T. A., Bigras, J.-L., et al. (2019). Can anthropometry and physical fitness testing explain physical activity levels in children and adolescents with obesity? J. Sci. Med. Sport t 23, 580-585. doi: 10.1016/j.jsams.2019.12.005

Riley, N., Lubans, D. R., Holmes, K., and Morgan, P. J. (2016). Findings from the EASY minds cluster randomized controlled trial?: evaluation of a physical activity integration program for mathematics in primary schools. J. Phys. Activ. Health 13, 198-206. doi: 10.1123/jpah.2015-0046

Ruiz-Ariza, A., Grao-Cruces, A., de Loureiro, N. E. M., and Martínez-López, E. J. (2017). Influence of physical fitness on cognitive and academic performance in adolescents: a systematic review from 2005-2015. Intern. Rev. Sport Exerc. Psychol. 10, 108-133. doi: 10.1080/1750984x.2016.1184699

Ruiz-Pérez, L. M., Ruiz-Amengual, A., and Linaza-Iglesias, J. L. (2016). Movimiento y lenguaje: análisis de las relaciones entre el desarrollo motor y del lenguaje en la infancia.[Movement and language: analysis of the relationships between motor and language development in children]. RICYDE Rev. Int. Cienc. Deporte 12, 382-397. doi: 10.5232/ricyde

Sallis, J. F., Buono, M. J., Roby, J. J., Micale, F. G., and Nelson, J. A. (1993a). Sevenday recall and other physical activity self-reports in children and adolescents. Med. Sci. Sports Exerc. 25, 99-108. doi: 10.1249/00005768-199301000-00014 
Sallis, J. F., McKenzie, T. L., and Alcaraz, J. E. (1993b). Habitual physical activity and health-related physical fitness in fourth-grade children. Am. J. Dis. Child. 147, 890-896. doi: 10.1001/archpedi.1993.02160320092025

Sardinha, L. B., Marques, A., Martins, S., Palmeira, A., and Minderico, C. (2014). Fitness, fatness, and academic performance in seventh-grade elementary school students. BMC Pediatr. 14:176. doi: 10.1186/1471-2431-14-176

Sardinha, L. B., Marques, A., Minderico, C., Palmeira, A., Martins, S., Santos, D. A., et al. (2016). Longitudinal relationship between cardiorespiratory fitness and academic achievement. Med. Sci. Sports Exerc. 48, 839-844. doi: 10.1249/MSS. 0000000000000830

Sember, V., Jurak, G., Kovaè, M., Morrison, S. A., and Starc, G. (2020b). Children's physical activity, academic performance and cognitive functioning: a systematic review and meta-analysis. Front. Public Health 8:307. doi: 10.3389/fpubh.2020. 00307

Sember, V., Jurak, G., Kovač, M., Đ*urić, S., and Starc, G. (2020a). Decline of physical activity in early adolescence: a 3-year cohort study. PLoS One 15:e0229305. doi: 10.1371/journal.pone.0229305

Sibley, B. A., and Etnier, J. L. (2003). The relationship between physical activity and cognition in children: a meta-analysis. Pediatr. Exerc. Sci. 15, 243-256.

Singh, A. S., Saliasi, E., Van Den Berg, V., Uijtdewilligen, L., De Groot, R. H. M., Jolles, J., et al. (2019). Effects of physical activity interventions on cognitive and academic performance in children and adolescents: a novel combination of a systematic review and recommendations from an expert panel. Br. J. Sports Med. 53, 640-647. doi: 10.1136/bjsports-2017-098136

Soric, M., Turkalj, M., Kucic, D., Marusic, I., Plavec, D., and Misigoj-Durakovic, M. (2013). Validation of a multi-sensor activity monitor for assessing sleep in children and adolescents. Sleep Med. 14, 201-205. doi: 10.1016/j.sleep.2012.11. 003

Stålesen, J., Vik, F. N., Hansen, B. H., and Berntsen, S. (2016). Comparison of three activity monitors for estimating sedentary time among children. BMC Sports Sci. Med. Rehabil. 8:2. doi: 10.1186/s13102-016-0028-y

Starc, G., Gril, M., and Cernilec, P. (2017). Academic performance of the most and least physically efficient children. Sodobna Pedagogika J. Contemp. Educ. Stud. 68, 130-144.

Starc, G., Kovaè, M., Strel, J., Buèar Pajek, M., Golja, P., Robiè, T., et al. (2015). The ACDSi 2014-a decennial study on adolescents' somatic, motor, psychosocial development and healthy lifestyle: Study protocol. Anthropol. Noteb. 21, 107123.

Tannehill, D., MacPhail, A., Walsh, J., and Woods, C. (2015). What young people say about physical activity: the Children's Sport participation and physical activity (CSPPA) study. Sport Educ. Soc. 20, 442-462. doi: 10.1080/13573322. 2013.784863
Tomkinson, G. R., Lang, J. J., Tremblay, M. S., Dale, M., LeBlanc, A. G., Belanger, K., et al. (2017). International normative $20 \mathrm{~m}$ shuttle run values from 1142026 children and youth representing 50 countries. Br. J. Sports Med. 51, 1545-1554. doi: 10.1136/bjsports-2016-095987

Tomporowski, P. D., Lambourne, K., and Okumura, M. S. (2011). Physical activity interventions and children's mental function: an introduction and overview. Prevent. Med. 52, S3-S9. doi: 10.1016/j.ypmed.2011.01.028

Torrijos-Niño, C., Martínez-Vizcaíno, V., Pardo-Guijarro, M. J., García-Prieto, J. C., Arias-Palencia, N. M., and Sánchez-López, M. (2014). Physical fitness, obesity, and academic achievement in schoolchildren. J. Pediatr. 165, 104-109. doi: 10.1016/j.jpeds.2014.02.041

Troiano, R. P., Berrigan, D., Dodd, K. W., Mâsse, L. C., Tilert, T., and Mcdowell, M. (2008). Physical activity in the United States measured by accelerometer. Med. Sci. Sports Exerc. 40, 181-188. doi: 10.1249/mss.0b013e31815a51b3

Van Dusen, D. P., Kelder, S. H., Kohl, H. W. III, Ranjit, N., and Perry, C. L. (2011). Associations of physical fitness and academic performance among schoolchildren. J. Sch. Health 81, 733-740. doi: 10.1111/j.1746-1561.2011. 00652.x

Wang, J. (2019). The association between physical fitness and physical activity among Chinese college students. J. Am. Coll. Health 67, 602-609. doi: 10.1080/ 07448481.2018 .1515747

Whitehead, M. (2010). Physical Literacy: Throughout the Lifecourse. Milton Park: Routledge.

Conflict of Interest: The authors declare that the research was conducted in the absence of any commercial or financial relationships that could be construed as a potential conflict of interest.

The handling editor declared a past co-authorship with one of the author VS.

Publisher's Note: All claims expressed in this article are solely those of the authors and do not necessarily represent those of their affiliated organizations, or those of the publisher, the editors and the reviewers. Any product that may be evaluated in this article, or claim that may be made by its manufacturer, is not guaranteed or endorsed by the publisher.

Copyright (c) 2022 Sember, Jurak, Starc and Morrison. This is an open-access article distributed under the terms of the Creative Commons Attribution License (CC BY). The use, distribution or reproduction in other forums is permitted, provided the original author(s) and the copyright owner(s) are credited and that the original publication in this journal is cited, in accordance with accepted academic practice. No use, distribution or reproduction is permitted which does not comply with these terms. 